\title{
Patient experiences and preferences for antiretroviral therapy service provision characteristics: implications for implementing differentiated service delivery in Northwest Ethiopia
}

Yihalem Abebe Belay ( $\nabla$ yih2000ho@gmail.com )

Debre Markos University

Mezgebu Yitayal

University of Gondar

Asmamaw Atnafu

University of Gondar

Fitalew Agimass Taye

Griffith University

\section{Research Article}

Keywords: Experience, preference, patients, antiretroviral therapy, Northwest Ethiopia

Posted Date: March 8th, 2022

DOI: https://doi.org/10.21203/rs.3.rs-1388885/v1

License: (c) (i) This work is licensed under a Creative Commons Attribution 4.0 International License.

Read Full License 


\section{Abstract}

\section{Background}

Understanding the experiences, needs, preferences, and behaviors of people living with HIV (PLHIV) are critical to tailor HIV treatment. However, there is limited research evidence in Ethiopia on the views of PLHIV regarding their positive and negative experiences on current antiretroviral therapy (ART) service and their preferred models of HIV treatment. Hence, this study aimed to explore patients' experiences of taking medications and preferences for ART service provision characteristics in Northwest Ethiopia.

\section{Methods}

This study with a phenomenological approach was conducted as part of the larger discrete choice experiment study. Purposive selection of stable and 18 years old and above PLHIVs using ART service at health facilities implementing four differentiated service delivery (DSD) models in East Gojjam, West Gojjam, Awi, and Bahir Dar special zones was done. Data were collected between July and September 2021. About 15 in-depth interviews with differentiated ART eligible patients were conducted. ATLAS.ti version 9 software was used for coding translated transcripts. A thematic analysis approach was employed.

\section{Findings}

Participants in this study had reported both positive and negative experiences in receiving ART. Five themes: stigma, time, availability of drug and provider, costs per clinic visit, provider-patient interaction were identified. Participants also reported varied preferences towards ART service features. Fifteen attributes: buddy system, ART refill (individualized or group), ART packaging and labeling, drug formulation and administration, ART room labeling, distance, location of service, preferences on involvement in treatment decision-making, person providing ART refills, provider's attitude, spatial arrangement of ART room, time of health facility operation, time spent at clinics and total cost of visit were identified.

\section{Conclusions}

Participants provided both positive and negative experiences with the aspects of current ART service. The preferences of PLHIVs towards ART service delivery features were heterogeneous. Policy and program efforts should tailor ART services that suit patients' needs and priorities in Ethiopia. Future research should also focus on understanding the satisfaction of patients enrolled among the four DSD models now under implementation in the country to get insights for optimizing each model implementation and to ensuring the strong quality of ART service provision for stable PLHIV.

\section{Background}


Global progress is being made towards achieving the Joint United Nations Programme on HIV/AIDS (UNAIDS) 95-95-95 HIV targets: 95\% of people with HIV diagnosed, 95\% of those diagnosed on treatment, and $95 \%$ of those on treatment virally suppressed [1]. Since 2015, the World Health Organization (WHO) has endorsed a differentiated service delivery(DSD) approach to providing HIV services to support reaching the UNAIDS targets by 2030 [2].

Differentiated service delivery -a "patient-centered approach that simplifies and adapts HIV services across the cascade to serve the needs of people living with HIV (PLHIV) better and reduce unnecessary burdens on the health system [3] "- has emerged as a core principle of HIV programs in resource-limited settings $[2,4,5]$. DSD is operationalized by adjusting the four building blocks: the frequency of visits, the location of service delivery, the type of health care workers, and the package of services according to the needs of different groups of PLHIV [6]. DSD models are broadly categorized as individual or group models, with service delivery at a facility or in the community [7].

DSD models for antiretroviral treatment (ART) for HIV are being scaled up throughout sub-Saharan Africa in the expectation that they will better meet the needs of patients, improve the quality and efficiency of treatment delivery and reduce costs while maintaining at least equivalent clinical outcomes [8]. Ethiopia has also adopted various DSD models since 2017 for stable PLHIV taking ART. Appointment spacing is being implemented in full scale. The health extension worker-managed community-based ART refill model has been piloted in Addis Ababa and Gambella since the end of 2018 and is being scaled up at six regions: Addis Ababa, Oromia, Amhara, Southern Nations, Nationalities and People, Sidama, and Gambella. Fast track ART refill and peer lead community ART refill models started in 2020 [9].

A successful client-centered HIV program aims to get antiretroviral medications into the hands of clients in a timely and efficient manner and to provide services that are convenient and welcoming. This starts with gathering insights to understand each client's experiences, needs, preferences, and behaviors, and then designing programs that reach them where they are and deliver services in ways that meet their needs which in turn support patients in adhering to ART as well as remain in the program for the long term $[10,11]$.

A previous systematic review found forgetting, lack of access to adequate food, stigma and discrimination, side effects, and being outside the house or traveling were barriers whereas social support, reminders, feeling better or healthier after taking ART, disclosing HIV status, and having a good relationship with a health provider were facilitators for patients' adherence to ART[12]. The previous metasynthesis also identified stigma and discrimination, fear of HIV status disclosure, task shifting to lay health workers, human resource and institutional challenges, mobile Health (mHealth), family and friend support, intensive case management, and, relationships with caregivers as facilitators or barriers for retention in HIV care[13]. A recent systematic review also found that patients preferred facility-based ART service over community service, shorter travel distance, reduced waiting time, less frequent clinic visits, good providers' attitude, having shared decision making, individualized models, and effective drugs with 
fewer side effects [14]. The same study also revealed that patients had negative values attached to the increased total cost of visits, HIV clinic branding, and current ART packaging.

Local research reporting patient perspectives on ART service and their preferences is critical in further program design of DSD models in Ethiopia which is a growing process. However, there is limited research evidence in Ethiopia on the views of PLHIV in the country regarding their positive and negative experiences on current ART service and their preferred models of HIV treatment. Therefore, this study aimed to explore patients' experiences of taking medications and preferences for ART service provision characteristics in Northwest Ethiopia to aid policymakers, program managers, and practitioners in Ethiopia as they implement and scale up DSD models.

\section{Methods}

\section{Study approach}

This qualitative study with the phenomenological design was conducted as part of the larger discrete choice experiment study. Data were collected between July and September 2021.

\section{Study setting}

The study was conducted in Bahir Dar special, Awi, West Gojjam, and East Gojjam Zones of Amhara National Regional State located in the northern part of Ethiopia. Currently, 343 health facilities are providing HIV treatment service to PLHIV in the region of which 41 of them implement four DSD models (appointment spacing, fast track ART refill, health extension, and peer-led community models). Similarly, there are 15 public health facilities ( 2 comprehensive specialized hospitals, 1 general hospital, 3 primary hospitals, and 9 health centers) implementing these models in the above-mentioned four Zones.

The study was conducted at the HIV clinics of four hospitals (Felegehiwot and Debre Markos comprehensive specialized hospitals, Finoteselam general hospital, and Dangila primary hospital) and two health centers (Debre Markos and Bichena). According to Ethiopia's health service structure of a three-tier system, the tertiary level of health care specialized hospitals serve 3.5 to 5.0 million people; the secondary level of care general hospitals serve 1 to 1.5 million people; primary level of care hospitals serve $60,000-10,000$ people and health centers serve $15,000-25000$ for rural and up to 40,000 for urban people [15].

\section{Sampling and recruitment}

A purposive selection of stable and 18 years old and above HIV positive individuals using HIV treatment service in the public health facilities of East Gojjam, West Gojjam, Awi, and Bahir Dar special zones was done. We recruited participants from different facilities in the sample based on a load of patients taking ART and facility type (tertiary, general and primary hospitals, and health centers). 
In-depth interviews with adults on ART who were virally suppressed and eligible for DSD [2] but not currently enrolled in one of the differentiated ART models were conducted. The facility staff members who were already aware of the study were requested to support the recruitment of patients from among those that were visiting for a regularly-scheduled ART appointment declared to be eligible to DSD models on the date of interview. The sample size was determined based on theoretical saturation, and 15 participants ( Felegehiwot hospital:5, Debre Markos hospital:3, Finoteselam hospital:2, Bichena hospital:1, Debre Markos health center:2, and Bichena health center:2) were involved.

\section{Data collection}

A semi-structured interview guide was developed and used for data collection. The guide consists of questions about the patients' experiences with current ART service and preferences towards features of ART service provision (Additional file 1). Interviews were conducted by the principal investigator (YAB) in the Amharic language. The interview guide was pre-tested among purposively selected participants at Debre Markos hospital before actual data collection. Data were collected from participants in a private room in the health facilities. The interviews were audio-recorded and lasted between 30 minutes and 105 minutes. Transcription of audio files and initial analyses were carried out in parallel with the data collection allowing the study principal investigator to get an insight into theoretical saturation. The interviewer wrote field notes during and after the interviews.

\section{Data analysis}

Participants' responses were transcribed verbatim. Then, the translated transcripts were imported into ATLAS. ti version 9 software for coding and were analyzed using a thematic analysis approach. Our analysis followed a hybrid approach of inductive and deductive coding. Initial deductive coding was based on the fixed topics from the literature review [14], study objectives, and interview guide followed by an inductive, data-driven generation of new codes. Familiarization with the data was done by reading and rereading the transcripts followed by developing the codebook. Memos were written during the coding process to capture impressions and to facilitate the identification of themes. Our study was reported according to the consolidated criteria for reporting qualitative studies[16] shown in Additional file 2.

\section{Trustworthiness of the study}

Trustworthiness in qualitative research is achieved by enhancing credibility, dependability, confirmability, and transferability[17]. The credibility of the study was enhanced by spending more time with participants in individual interviews until data saturation was reached. There was also iterative questioning (the use of probes to elicit detailed data and returning to matters previously rose by an informant and extracted related data through rephrased questions) to enhance credibility. Dependability was enhanced by maintaining an audit trail through keeping all copies of notes, transcribed and recorded data for future use, including supplying participants with researchers' personal and academic information for contact or explanation at any time. 
Confirmability was enhanced by conducting a pilot study, which served as a pre-test to the interview schedule, and interviewing skills from 3 participants at Debre Markos hospital helped to refine the study methods. The results of the pilot were not a part of the report presented in the final study. Transferability was enhanced using a non-probability purposive sampling method to collect data from participants. We also supported transferability via rich descriptions and verbatim quotations from the transcripts.

\section{Ethical approval}

This study received ethical approval from the Institutional Review Board of the University of Gondar (approval number V/P/RCS/05/762/2021). A formal letter obtained from Amhara Public Health Institute was given to each health facility to get permission and cooperation. After briefing the purpose of the study, written informed consent was taken from each participant before the data collection.

\section{Findings}

\section{Participant characteristics}

A total of 15 PLHIV who use ART were interviewed. The mean age of participants was 38.8 years $(S D=3.59)$. The mean duration of ART intake was 10.1 years $(S D=1.43)$. About two-thirds were female, nearly a quarter were married, $46.7 \%$ had not attended education and $40 \%$ attended secondary and higher education. Moreover, about two-thirds were employed (Table 1).

Key qualitative findings are described below and organized according to the two major themes associated with the patients' experiences with ART service and preferences for ART service delivery characteristics expressed across all participants. An overview of patients' experiences with ART service and their preferences for ART service provision characteristics identified from the interview are presented in Tables 2 and 3 respectively. Illustrative comments from interviewed participants are included as appropriate.

\section{Participant experiences with antiretroviral therapy service}

Participants in this study had reported both positive and negative experiences when they received care or treatment from the ART clinics.Stigma, time (waiting, facility opening, travel, frequency of health facility visit and time convenience with school and work), drug and provider availability, costs per clinic visit, and provider-patient interaction were the themes that emerged regarding the participants' experiences with ART and are highlighted in the following section as follows:

\section{Stigma}

Perceived/anticipated stigma following HIV status disclosure was quite prevalent among all PLHIV in the study. Maintaining secrecy or limiting disclosure of HIV status appeared to be a protective strategy among many participants. 
"I take my drug in a hiding place when my child sleeps since she didn't know about my status. I always come from a remote area which takes 1 hour by bus since there is still a stigma in our community. My social life and work could be affected if I get service in my locality". (33 years old female)

"No one knows about my HIV status except my wife hence I didn't experience stigma from others". (56 years old male)

Some participants reported experiences of stigma from family, community, and healthcare settings.

"My husband's family members were treating me negatively since they considered me as doing some evil activity on him. They advised him to stop the HIV drug and rather go to a traditional healer". (57 years old female)

"I have an experience of discrimination by a woman with a rental dorm. She refused to accept me to rent in her house as a result of knowing my HIV status". (27 years old female)

"I have found a discriminatory action by the gatekeepers. They [gatekeepers] openly asked me the reason for the entry to the hospital in front of many people requesting to enter the hospital during COVID-19 movement restriction". (40 years old male)

However, no participant mentioned an experience of stigma by their healthcare providers.

\section{Time}

The participants in this study have mentioned their positive and negative experiences about waiting time, facility opening, travel time, frequency of health facility visits, and time convenience with school and work.

Waiting time. Many participants reported large crowds of clients at the ART clinics in the past. The large crowds in their opinion resulted in long waiting hours before consultations or drug refills.

"I wait 5-6 hours in this hospital to get the ART service. The providers may even postpone our turn to the afternoon time". (40 years old female)

"It takes me 5 minutes of travel on foot. The main problem is the long waiting time in the health center. It takes 1-2 hours to wait here depending on the number of clients being served. We are getting our cards from the main card room with other HIV-negative individuals creating a big concern for us". (38 years old female)

Some participants however reported waiting less time at a health facility.

"We do not wait for more here. There is a special service room in this hospital for ART service. There are many providers in each room of ART service". (57 years old female) 
Facility operation time. Clinic opening and closing hours were frequently mentioned as frustrating patients' access to care.

"It could be okay to return to work if we get the service before 2:30 in the morning. But the providers are losing our time here by starting the service at 3:00 instead of the legal 2:30". (40 years old male)

"I have faced a challenge to get my drugs when I came here after 10:00 in the afternoon despite the work time being until 11:30 from Monday to Friday". (40 years old male)

One participant, however, said that the providers start working early in the hospital.

"The providers enter the hospital early and they serve us at a speed". (32 years old female)

Travel time. Clients reported varied experience in the duration of travel time from home to a facility. Many participants highlighted less travel time from home to a health facility.

"It takes me about 30 minutes to travel from home to this facility on foot". (22 years old male)

Some participants reported more travel time from home to a facility.

"I come from a remote area which takes 1 hour by bus due to continued stigma in our community". (33 years old female)

Frequency of facility visit. Clients mentioned their experience of more frequency of health facility visit schedules in the past.

"I was visiting the facility every month for 7 years". (22 years old male)

Other participants however reported less frequent visits.

"It is not difficult to come to this hospital since I return here every 3 months schedule". (33 years old female)

Time convenience. Some participants reported time inconvenience with school and work.

"The laboratory test clashes with my education schedule since the provider always tell me to come in the morning where there may be a class in the school". (22 years old female)

"I am still frustrated with disclosing my status to others. I have tried to request one of the providers here and she sent me my drugs via postal service since I was not able to come on the appointment date due to a clash with my work". (33 years old female)

\section{Drug and provider availability}

No respondents in this study reported experiencing a complete ARV drug stock-out at the health facilities. 
"I am satisfied with the drug supply in this hospital". (40 years old female)

Most participants reported a shortage of health care providers in the ART clinics.

"There should be additional providers employed in the ART room since the number of clients is many hence to avoid waiting for a long time here". (22 years old female)

On the other hand, one participant mentioned the availability of adequate providers in the ART rooms of the hospital.

"There are many providers in each room of ART service in the hospital". (57 years old female)

\section{Costs per clinic visit}

The participants in this study mentioned that only ARV medication and a few laboratory investigations were free. The participants highlighted that they cover the costs of transportation, drugs for opportunistic infections, and additional costs including food.

"Since I am near the hospital, I do not pay much money to come here. The HIV drug is free. If there are no drugs in the hospital other than HIV drugs, we pay for that". (25 years old female)

Some participants expressed concern about the expensive cost of transportation and accommodation when coming from remote areas due to fear of stigma and discrimination in the community.

"I come from a remote area which takes 1 hour by bus with 60 Birr (\$1.5) cost. Since there is still stigma in our community, I am forced to come here for getting ART service. I may take paying fewer costs for a taxi if I attended in my locality". (33 years old female)

"I pay for the transport and food costs when I come here. But, I did not pay for drugs at this hospital since I have no opportunistic infections". (40 years old male)

Participants reported that they miss work when seeking care.

"I have discussed with my providers to give me drugs with additional stock at least for 10 days in addition to the usual prescription to avoid work inconvenience". (33 years old female)

\section{Provider-patient interaction}

Encouragement: Most participants mentioned the good attitudes and behaviors of healthcare workers towards them, which they said have encouraged them to continue accessing HIV care and treatment.

"The providers have patience and provide service in a good manner. They counsel us when we miss care. They do not anger on us. They serve us in love". (22 years old male) 
"The providers are kind enough to treat us. Some providers have HIV and help us properly. They [providers with HIV] counsel us in a good manner. It is like knowing about the hungry status of someone by remembering their own hungry experience. I always want to contact them [providers with HIV]". (33 years old female)

Discouragement. Some patients reported encountering HCWs who were disrespectful, non-caring, and providing inadequate counseling.

"The previous workers in the ART room were very good to follow us closely and get us treated if we become sick. But now, the providers are not careful enough to treat us properly. They [current providers] did not care while I slept on the bench in the waiting room here in the past". (27 years old female) In terms of counseling, the providers at this hospital are relatively less attentive to us compared to the health center although they (providers) ask about our progress and proper drug-taking". (40 years old female)

\section{Participant preferences for antiretroviral therapy service characteristics}

Fifteen attributes (themes) with respective attribute levels were identified in the thematic analysis. Attributes and attribute levels were identified from the transcripts and prominent participants' quotes were directly extracted to illustrate each attribute and attribute level (Table 3). The identified attributes are highlighted in the following section.

\section{Buddy system}

The majority of participants preferred having physical contact with the health facility over having someone assisting in collecting their pills. The reasons cited were the need for health status check, weight check, counseling, checking the effectiveness of taking the drug, and lack of trust in someone collecting drugs.

"It is beneficial if I come here myself, asked about my health status and get weight check and counseling from the providers about drug-taking, feeding and avoiding drinking alcohol". (57 years old female)

"I prefer to come physically here to be checked about my health status and whether the drug is working or not. It should not be thought of simply taking drugs from here". (25 years old female)

"I prefer to take drugs myself since I will not be happy if another person brings drugs for me that may not be trusted like me". (40 years old male)

There were however some participants who preferred someone to take drugs for them from the facilityon their behalf in case of time inconvenience on date of appointment, bedridden condition, and engage in their own work activities on the facility visit days. 
"I prefer having the other person to assist me in drug-taking since there may be some time inconvenience for me to come here". (33 years old female)

"It would be better to have other persons who assist in case of bedridden or paralyzed cases". (72 years old male)

\section{Individualized or group ART refill service}

Themajority of the participants have preferred individualized ART refill service to group-based service due to a need for maintaining privacy and confidentiality, avoiding clashing with other group members, weight check-ups, and fear of drug change and getting appropriate service.

"I prefer the individualized service to avoid clashing with other group members regarding the scheduled time to meet". (72 years old male)

"Wow! I prefer individualized service since there may be a change of drugs or no opportunity to have weight measured". (27 years old female)

"I prefer the individual-based service since there may not be appropriate service in a group-based approach". (40 years old female)

"I prefer the individual-based service to avoid disclosing my status when I form groups and take drugs together with the other clients". (40 years old male)

Some participants on the other hand chose the group form of ART service for reasons of experience sharing, strengthening social relationships, and using the time for work.

"I prefer the group-based service since we have an opportunity of sharing ideas". (22 years old male)

"I prefer the group-based service since it helps us to support one another by strengthening our social interactions. It also saves our time to come here individually and use our time for our works by taking drugs in the village". (40 years old female)

\section{ART packaging and labeling of drug package}

Nearly all participants indicated that they would prefer a change of drug package because the box and bottle are large and easily identifiable by others.

"I also expect that the drug package should be changed since the current bottle-based package created discrimination by others as they could easily identify it. The bottle should be changed so that the container can handle many drugs and even we can put it in our pocket to avoid direct advertising act of the current package. (33 years old female)

Regarding the labeling of drug packages, the majority of participants preferred the medicine labels must not be clear to avoid disclosure of HIV status. 
"I think there should not be the labeling of the drug package to avoid being readable by other people than me like my child. She may search via Google and know about her status". (33 years old female)

Some preferred however the medicine labels must be clear for easy identification by clients.

"I don't care about the labeling of drug package. There may be some others who may discriminate us [clients with HIV on ART] when they [others] see the package and read the labeling of ARV drugs on the boxes". (38 years old female)

\section{Drug formulation and administration}

There was heterogeneity of participants' preferences for the aspects of drug formulation and administration. Some participants preferred the ARV drug formulation with additives and/protein.

"I would be happy if there is a curable drug for us or drugs with fewer side effects or some additives in the drug that boosts the client's immunity like vitamins". (33 years old female)

"I expect there is a drug that builds our body like proteins in the drug so that you will be fat and similar to other HIV naïve people". (40 years old male)

Some participants preferred the effective or curable drug formulation.

"If possible I prefer if there is a permanent cure for HIV. I would stop visiting this hospital if there is a cure for HIV". (38 years old male)

Some participants preferred an injectable form of ARV drugs.

"It could be better if there is a vaccination like for the other diseases or an injection that could be used at least for one year". (25 years old female)

\section{ART room labeling}

The majority of participants preferred the clear labeling of the ART room to help them in easy identifying of service delivery room.

"I prefer the clear labeling of the ART room to help me in identifying the service delivery room". (72 years old male)

Some of the participants preferred the non- labeling of the ART room to avoid notifying them of HIV status.

"I prefer the non-posting of the room to avoid discrimination by others while I get into this service room". (22 years old male)

\section{Distance from residence to a clinic}


Most of the participants preferred a near distance from their homes to the health facilities.

"I prefer the clients get the service in a near place. It reduces time, costs for transportation and could help to engage in other work activities". (38 years old male)

Some however preferred a far distance from home to health facility due to the concerns of privacy and confidentiality.

"It is my interest to come from a remote place since there is still a stigma in our community while getting service in my locality". (33 years old female)

\section{Frequency of receiving ART refills}

The participants of this study have varied preferences for the frequency of receiving ART refills. The majority of them preferred having a facility visit every 6 months for ART refills.

"I prefer to come every 6 months per year. But we can come here if we become sick in between the appointment dates". (38 years old female)

Some participants chose to attend the health facilities every 3 months.

"I prefer to come every three months here. It could help me to be checked about my health status regularly. If I take the drugs every 6 months or yearly, I may be sick with an opportunistic infection and my viral load could be increased due to the long time to check my status by the providers". (57 years old female)

Some of the participants preferred yearly visit for ART refills.

"I prefer to come once per year if I am healthy. It avoids transport costs and losing our daily works there at our locality. I may come at any time here if I have illness in between". (32 years old female)

\section{Location of ART service}

The majority of the participants preferred a facility-based ART service over the community-based service including home delivery. The common reasons mentioned were concern on confidentiality, getting health investigation, getting timely and appropriate service.

"I prefer the facility-based service since there may be a problem that will occur to give service at the community level by the current level of understanding. Providers may break confidentiality to let know others know about my status. ". (33 years old female)

"I prefer to get the service by coming to the hospital since / could get investigations done if I feel sick". (22 years old female) 
"I prefer the facility-based ART service since we could get our providers in time and get appropriate service here. I have a concern there in the community that the providers may not deliver service like the providers in the facility. I may not be at home on the appointment date or I may be not aware of the exact appointment date there and I may create a problem for my providers in this case". (72 years old male)

Some participants chose the community-based ART service due to reasons of time-saving, clients knowing each other, and avoiding long queues at a health facility.

"I prefer the community-based service since it [community-based service] saves time and lets clients know each other". (57 years old female)

"I would be happy if the service is given at the community level since it avoids a long queue at this hospital and waiting time". (40 years old female)

\section{Preferences on involvement in treatment decision-making}

Some participants preferred the provider's entire treatment decision on their behalf.

"I prefer to select the model of my choice since I have a reason to choose from alternatives depending on my context. The provider should not decide for me". (72 years old male)

Some participants preferred the provider's entire treatment decision on behalf of them.

"I couldn't decide my model of choice. The provider should select the appropriate option since he knows the benefits and harms of this approach. The clients shouldn't select the options for them rather the providers should select them". (40 years old male)

Some other participants attached more importance to reaching a consensus and having a shared responsibility in decision-making.

"I prefer a joint decision to select the model. There could be sharing of each idea by the clients and the providers. There should be an agreement between the two entities. There may be damage if one of the two simply selects the model". (25 years old female)

\section{The person providing ART refills}

Most of the study participants preferred receiving the ART refills by the healthcare workers at the health facility due to their knowledge, training, and concern on confidentiality.

"I prefer the healthcare workers since they have their training. They [health care workers] can give the drugs by knowing the benefits and the harms. But in the case of the HEWs or peer leaders, they [HEWs or peer leaders] lacked the appropriate knowledge and even they may give the drugs by exchanging our drugs. I never trust them in this regard". (27 years old female) 
"I prefer the healthcare workers since they are trained to identify and manage the problems that I may have by critically evaluating my health status. However, the peer leaders are similar to me in terms of knowledge and couldn't provide drugs for me properly". (38 years old male)

"I prefer the healthcare workers to deliver the ART service. I don't accept the peer leaders distributing our drugs since there is discrimination by the local community perceiving us having a meeting of HIV-positive people in the community". (40 years old female)

One participant chose to get the ART refill by the peers for sake of being understood well.

"I prefer the peer leaders to bring us our drugs since they know everything and they have experience of drug-taking. They [peer leaders] give more empathy to us compared to health extension and health care workers. Others [health extension and health care workers] couldn't appreciate the context despite they have been trained on the disease and the drug and give the service by reading on drug". (40 years old female)

One participant preferred the HEWs for concern of maintaining privacy.

"I prefer the health extension worker to give me drugs since I can go to her [health extension worker] without notifying my status". (40 years old male)

\section{Provider's attitude}

All participants in this study put a strong preference for having nice over rude providers. They valued more on the empathy and positive attitude of their providers.

"My choice depends on the provider you get every visit. I had one female provider who treats me with a bright face and want others [providers] to treat me like her". (38 years old male)

\section{The spatial arrangement of the ART room}

Most participants preferred a separated ART clinic from main health facility buildings for sake of privacy.

"I prefer the separate building of the ART room to avoid the associated stigma if the ART room is available with other service rooms of the health facility". (72 years old male)

Some participants however prefer the shared space of services in the main health facility buildings for reasons of hiding HIV status and considering HIV service similar to other services.

"I prefer the service should be given with another service in the same room to avoid HIV status. The providers should treat them accordingly based on the clients' situation instead of a separate service for $A R T^{\prime \prime}$. (33 years old female)

"I would be okay if the service is connected with other services. It is similar to other services in the hospital. I have raised a question for myself why the service room is isolated from other service delivery 
rooms in the same facility". (22 years old female)

\section{Time of the health facility operation}

The majority of participants in this study preferred getting ART service in the workweek (Monday to Friday) in the usual hours (2:30-6:30 morning and 7:30-11:30 afternoon).

"I prefer the usual working days and hours since it is enough to get the service at that time". (38 years old female)

Some preferred to get the service in the work week with weekend days.

"I prefer the extra facility opening time especially Saturday and Sunday in addition to the usual working days and hours. I am not comfortable with the facility openings before 2:30 in the morning and after 11:30 in the afternoon since we do not come to this hospital at such time". (40 years old female)

Others chose to obtain the service 24 hours of the day at any time of the week.

"I choose a 24 hour ART service in this hospital since we may be sick at any time. We get the service in case of difficulty if the facility is opened all days including night". (25 years old female)

One participant preferred weekend service only.

"I prefer the weekend-based service since I come here freely to take drugs on these days". (22 years old female)

\section{Time spent at clinics in ART pick-ups}

Most study participants preferred less waiting time at the ART room in ART pick-ups including registration, consultation, and pharmacy dispensing, cited saving time for work or school as the main factor.

"I would prefer to get service in the shortest time since I am busy either going to work or school. But, if the service demands to wait for more, I would wait here instead". (22 years old male)

Few participants however chose to wait more time in the facility due to a need for adequate time to discuss with providers and talk with peers in the facility.

"I prefer to wait a long time to learn from my providers about the drug and the related things. I also want to talk with other similar clients in this hospital. I would be happy when I come here and see other clients taking drugs like me. I become frustrated when I am alone in my home". (40 years old female)

\section{The total cost of the visit}


Nearly all participants preferred either a free or subsidized cost of the visit including transportation and medications other than ARV drugs.

"I prefer the service free of charge. There should be a special arrangement from the facility to cover the cost of transportation for those clients who do not generate income like old persons". (25 years old female)

"I believe that the free service that is currently being done is a good one. I recommend a balanced payment or a free service for those clients unable to pay for it [service]". (33 years old female)

One participant however was willing to pay for additional cost of transportation.

"I will pay whatever transport cost I have been requested to come here. I have health insurance and do not pay other payments in this hospital". (40 years old female)

\section{Discussion}

In this study, we explored ART service experiences and preferences among stable adult people living with HIV in a multi-site context in Northwest Ethiopia. The study provides evidence of key characteristics of a positive or negative patient treatment experience and the heterogeneous preferences on the key features of ART service provision.

\section{Participant experiences with antiretroviral therapy service}

Participant interviews revealed that HIV stigma persists. The main type of stigma evident among our respondents was anticipated stigma where they fear facing negative outcomes from others following HIV status disclosure. This finding is consistent with other recent research linking HIV stigma to low disclosure $[18,19]$. This type of stigma has implications for service use and ART adherence. Beyond perceived stigma, participants reported experiences of stigma (being rejected by family, denial of house renting, and being asked to notify their status openly at hospital entrance). This suggests the need for strengthening social and behavior change communication interventions at different levels to achieve the UNAIDS's vision of zero discrimination towards people living with HIV by 2030[20].

Participants reported long waiting times in health facilities before consultations or drug refills in the past due to overcrowding with many HIV-positive patients. This implies the need of adopting and implementing DSD to avoid congestion of health facilities and manage patients efficiently. Similarly, participants stated a negative experience with clinic operation time, particularly the late opening and early closing times that made frustrating their access to care. This suggests the need for continuity of service throughout all times of the day. Concerning travel time, participants reported having less travel time from home to health facility except for some participants who came from remote areas fearing stigma to receiving service in their locality. This is contrary to the finding of a previous study in Uganda [21]. Participants mentioned their experience of more frequent health facility visit schedules in the past. This 
has an important implication for implementing the DSD approach to reduce the frequency of health facility visits for clients.

Regarding provider-patient interaction and communication, participants stated positive and negative attitudes of health care workers when receiving services. Good attitudes and behaviors of healthcare workers encouraged patients to continue accessing HIV care and treatment. This has an important policy implication for ensuring the availability of a motivated, competent, and compassionate health workforce.

Participants stated financial concerns about costs of transportation, drugs for opportunistic infections, additional costs including food and accommodation as well as missed work when seeking care. This is comparable with a study conducted in Iran [22]. This highlights that further decentralization of ART services to lower-level health facilities and availing a free or subsidized cost for treatment of opportunistic infections are needed which in turn contributes towards the achievement of the goal of universal health coverage by 2030 .

Participants reported a shortage of health care providers in the ART clinics about the number of clients getting service there causing long waiting times. This has an important implication for more investment in human resources for health thereby improving the quality of ART service provided to patients in the health facilities. On the other hand, respondents had positive experiences in drug availability at the HIV clinics. This highlights that health facilities had given much attention to consistent ARV drug supply.

\section{Participant preferences for antiretroviral therapy service characteristics}

Regarding the buddy ART collection system, participants had varied preferences for having someone supporting to take drugs from the facility on their behalf in case of difficulty. The majority of them chose to collect ARVs themselves whereas some respondents preferred having a support person in the family or a friend assisting to pick up drugs from facilities. This is consistent with a study conducted in Zambia [23]. This suggests HIV-care providers need to encourage the involvement of a treatment supporter for individuals taking ART long-term.

Participants put varied values on individualized versus group-based ART refills. The majority attached great importance to the individualized service mainly related to concerns of privacy and confidentiality. This finding is in agreement with studies conducted in Zimbabwe[24] and in Kenya [25]. This has an important implication of practical challenge in implementing and expanding the DSD models in Ethiopia.

Concerning ART packaging and the labeling of drug packages, participants valued more the need for change in large boxes and bottle-based packages, and the avoidance of labeling the drug package as ARV for sake of decreasing the associated stigma. This is consistent with a study in Tanzania [26]. This suggests relevant implications for the need for innovative ART packaging to improve ART adherence and achieve viral load suppression. Similarly, participants preferred a change in the existing drug formulation and administration towards ARV drugs with additives and/protein, effective or curable drug formulation, 
injectable form, and with fewer side effects. This is comparable with a study conducted in the United States [27]. This has an important implication for future drug development initiatives to invest more in novel drug discovery.

Regarding the spatial arrangement of the ART room, most participants highly valued a separated ART clinic from main health facility buildings for privacy concerns. Some participants however prefer the integrated services in the main health facility buildings for the reason of hiding HIV status. This is in line with a study conducted in both Zambia and South Africa [28]. This has policy implications to enact PLHIV-friendly policies in HIV spatial organization to diminish the related stigma. In connection with this, most participants preferred the clear labeling of the ART room to help them in easy identifying the service delivery room in the presence of some other respondents who chose non-labeling of the ART room to avoid notifying their HIV status. This is consistent with a study conducted in South Africa where the probability of choosing to attend a clinic decreases if the clinic was branded as an HIV clinic [29]. This suggests that clinic branding may constitute important barriers to ART uptake and adherence which needs policy intervention.

Most of the participants preferred a near distance from their homes to the health facilities. This finding is in line with previous studies [30,31]. This highlights a need for further decentralization of ART service to where people live and work to achieve universal access to antiretroviral treatment. Regarding the frequency of health facility visits, participants chose to have a facility visit every 6 months for ART refills. This finding is consistent with a study conducted in Kenya [25]. This suggests that multi-month scripting best aligns with patients' preferences, an insight that can help prioritize use of different DSD models in Ethiopia. Regarding the location of service delivery, the majority of the participants preferred a health facility-based ART service over a community-based service. This is in line with an earlier study [25]. This highlights an important implication to enhance the promotion of the community-based models as an alternative to the facility-based models hence reducing overloading of the existing facilities with clinically stable patients.

Participants' preferences for their involvement in treatment decision-making were varied. The majority of them preferred to make all final decisions alone. Some preferred that their doctor make all or most decisions and others preferred to share decisions with their doctor. This is comparable with a study conducted in the United States [32]. This highlights the need for communication about patients' expectations, wishes, and preferences for participation in upcoming HIV treatment decisions.

Regarding the person providing ART refills, most participants preferred receiving the ART refills by the healthcare workers at health facilities than health extension workers or peers. This is in line with a study conducted in Kenya [25]. This has an important implication for providers, health system administrators, health workforce planners, and policymakers to better understand patient perspectives and design care that enhances patient satisfaction.

Participants preferred getting ART service in workweek and usual hours; work week with weekend days or 24 hours of the day at any time of the week. This is consistent with a study conducted in Zambia [33]. 
This has policy implications on the health workforce and other resource allocation to increase service availability beyond the standard routine practice hours and days.

Participants in this study put a strong preference for nice over rude providers. This is in line with a study in Zambia [33]. This highlights enhancing policy interventions on the implementation of compassionate, respectful, and caring human resources for health.

Participants preferred less waiting time at the ART room in ART pick-ups. This finding is consistent with a study in Ghana[34]. This highlights a policy intervention to increase the number of health personnel at ART clinics to reduce the time HIV-positive patients spend at such facilities. Similarly, participants preferred either a free or subsidized cost of the visit including transportation and medications other than ARV drugs. This is consistent with a previous study [14]. This has policy implications for increased access to ART service to where people live and work as well as insurance coverage.

\section{Limitations}

This study was subject to limitations. First, it is not possible to generalize the findings of this qualitative study due to its relatively small sample size. Second, we do not have data about the actual experiences or preferences of patients since these findings are based on what the interviewed participants chose to report to the interviewer. Third, the responses from the participants of this study may not be reflective of all PLHIV taking ART since we only included a group of patients attending health services in the selected sites.

\section{Conclusions}

HIV-positive patients provided both positive and negative experiences with the aspects of current ART service delivery in their interactions with the health system and clinical environment. The preferences of PLHIVs towards ART service delivery features were heterogeneous. Therefore, policy and program efforts aimed at achieving optimal enrolment, retention in care, and viral suppression should tailor ART services that suit patients' needs and priorities in Ethiopia. Future research should also focus on understanding the satisfaction of patients enrolled among the four DSD models (appointment spacing, fast track refill, peer-led and health extension professional-led) now under implementation in the country to get insights for optimizing each model implementation and to ensuring the strong quality of ART service provision for stable PLHIV.

\section{Abbreviations}

AIDS

Acquired Immunodeficiency Syndrome

ART

Antiretroviral Therapy

ARV 
Antiretroviral

DSD

Differentiated Service Delivery

$\mathrm{HCW}$

Health Care Worker

HEW

Health Extension Worker

HIV

Human Immunodeficiency Virus

PLHIV

people living with HIV

SD

Standard Deviation

UNAIDS

The Joint United Nations Programme on HIV/AIDS

WHO

World Health Organization

\section{Declarations}

Ethics approval and consent to participate: This study obtained ethical approval from the Institutional Review Board of the University of Gondar and a formal letter obtained from Amhara Public Health Institute. Participants provided written informed consent documenting willingness to participate in interviews and to have the audio recorded.

Consent for publication: Not applicable.

Availability of data and materials: The datasets used during the current study are available from the corresponding author on a reasonable request.

Competing interests: The authors declare that they have no competing interests.

Funding: This study was supported by the University of Gondar in Ethiopia.

Authors' contributions: All authors participated in the conception and design of this project including data collection tool development. YAB collected and analyzed interview data. YAB drafted the manuscript. Feedback and editing were provided by MY, AA, and FAT. All authors read and approved the final manuscript.

Acknowledgments: We acknowledge the University of Gondar for funding this study. The authors wish to acknowledge the study participants for their willingness to share their opinions. The authors would also 
like to acknowledge the clinical staff at the participating health care facilities who assisted with recruitment.

Authors' information: Not applicable.

\section{References}

1. Joint United Nations Programme on HIV/AIDS: 90-90-90: an ambitious treatment target to help end the AIDS epidemic. Geneva: Unaids 2014.

2. World Health Organization: Consolidated guidelines on the use of antiretroviral drugs for treating and preventing HIV infection: recommendations for a public health approach: World Health Organization; 2016.

3. Grimsrud A, Bygrave H, Doherty M, Ehrenkranz P, Ellman T, Ferris R, Ford N, Killingo B, Mabote L, Mansell TJJotIAS: Reimagining HIV service delivery: the role of differentiated care from prevention to suppression. 2016, 19(1).

4. PEPFAR FY. 2020 COP Guidance for All PEPFAR Countries. Available at: https://www.state.gov/wpcontent/uploads/2020/01/COP20-Guidance_Final-1-15-2020.pdf. Accessed Jan 27, 2022.

5. The Global Fund to Fight HIV TaM: HIV Information Note. Available at:

https://www.theglobalfund.org/media/4765/core_hiv_infonote_en.pdf. Accessed January 9, 2022. 2019.

6. Society IA: Differentiated care for HIV: a decision framework for antiretroviral therapy delivery. In.: International AIDS Society Durban, South Africa; 2016.

7. Grimsrud A, Barnabas RV, Ehrenkranz P, Ford NJJotIAS: Evidence for scale up: the differentiated care research agenda. 2017, 20(Suppl 4).

8. Long L, Kuchukhidze S, Pascoe S, Nichols BE, Fox MP, Cele R, Govathson C, Huber AN, Flynn D, Rosen SJJotIAS: Retention in care and viral suppression in differentiated service delivery models for HIV treatment delivery in sub-Saharan Africa: a rapid systematic review. 2020, 23(11):e25640.

9. Ethiopia Country Operational Plan COP 2021 Strategic Direction Summary. Available from https://www.state.gov/wp-content/uploads/2021/09/Ethiopia_SDS_Final-Public_Aug-11-2021.pdf. Accessed on February 12022.

10. Client-Centered HIV Services Fact Sheet. Available from https://www.state.gov/wpcontent/uploads/2020/07/PEPFAR_Client-Centered-HIV-Services_Fact-Sheet_2020.pdf. Accessed on February 2, 2022

11. Yoder PS, Mkhize S, Nzimande SJHsT, South Africa: Patient Experiences In Antiretroviral Therapy Programmes In KwaZulu-Natal, South Africa. 2009.

12. Croome N, Ahluwalia M, Hughes LD, Abas MJA: Patient-reported barriers and facilitators to antiretroviral adherence in sub-Saharan Africa. 2017, 31(7):995. 
13. Hall BJ, Sou K-L, Beanland R, Lacky M, Tso LS, Ma Q, Doherty M, Tucker JDJA, Behavior: Barriers and facilitators to interventions improving retention in HIV care: a qualitative evidence meta-synthesis. 2017, 21(6):1755-1767.

14. Belay YA, Yitayal M, Atnafu A, Taye FAJCE, Allocation R: Patients' preferences for antiretroviral therapy service provision: a systematic review. 2021, 19(1):1-25.

15. PRIMARY HEALTH CARE SYSTEMS: Case study from Ethiopia. Retrieved from https://www.who.int/alliance-hpsr/projects/alliancehpsr_ethiopiaabridgedprimasys.pdf?ua=1

16. Tong A, Sainsbury P, Craig JJljfqihc: Consolidated criteria for reporting qualitative research (COREQ): a 32-item checklist for interviews and focus groups. 2007, 19(6):349-357.

17. Pilot D, Beck C: Nursing research: Generating and assessing evidence for nursing practice. In.: Wolters Kluwer| Lippincott Williams \& Wilkins. https://schol ar. google. com ...; 2012.

18. Emlet CAJApc, STDs: Experiences of stigma in older adults living with HIV/AIDS: A mixed-methods analysis. 2007, 21(10):740-752.

19. Karamouzian M, Akbari M, Haghdoost A-A, Setayesh H, Zolala FJJotAoNiAC: "I am dead to them": HIV-related stigma experienced by people living with HIV in Kerman, Iran. 2015, 26(1):46-56.

20. Geneva. UJU: Understanding fast-track: accelerating action to end the AIDS epidemic by 2030. 2015.

21. Bajunirwe F, Tumwebaze F, Akakimpa D, Kityo C, Mugyenyi P, Abongomera GJBri: Towards 90-90-90 target: factors influencing availability, access, and utilization of HIV services-a qualitative study in 19 Ugandan districts. 2018, 2018.

22. Asadi H, Imani-Nasab M-H, Garavand A, Hasoumi M, Kia AA, Haghi B, Setoodehzadeh FJTOAJ: HIV positive patients' experience of receiving health care services: A phenomenology study in Iran. 2018, 12(1).

23. Eshun-Wilson I, Mukumbwa-Mwenechanya M, Kim H-Y, Zannolini A, Mwamba CP, Dowdy D, Kalunkumya E, Lumpa M, Beres LK, Roy MJJoaids: Differentiated care preferences of stable patients on antiretroviral therapy in Zambia: a discrete choice experiment. 2019, 81(5):540.

24. Rabkin M, Strauss M, Mantell JE, Mapingure M, Masvawure TB, Lamb MR, Zech JM, Musuka G, Chingombe I, Msukwa MJPo: Optimizing differentiated treatment models for people living with HIV in urban Zimbabwe: findings from a mixed methods study. 2020, 15(1):e0228148.

25. Dommaraju S, Hagey J, Odeny TA, Okaka S, Kadima J, Bukusi EA, Cohen CR, Kwena Z, Eshun-Wilson I, Geng EJPo: Preferences of people living with HIV for differentiated care models in Kenya: A discrete choice experiment. 2021, 16(8):e0255650.

26. Muiruri C, Jazowski SA, Semvua SK, Karia FP, Knettel BA, Zullig LL, Ramadhani HO, Mmbaga BT, Bartlett JA, Bosworth HBJPp et al: Does antiretroviral therapy packaging matter? Perceptions and preferences of antiretroviral therapy packaging for people living with HIV in northern Tanzania. 2020, 14:153.

27. Eaton EF, McDavid C, Banasiewicz MK, Mugavero MJ, Knight SJJPp, adherence: Patient preferences for antiretroviral therapy: effectiveness, quality of life, access and novel delivery methods. 2017, $11: 1585$. 
28. Bond V, Nomsenge S, Mwamba M, Ziba D, Birch A, Mubekapi-Musadaidzwa C, Vanqa N, Viljoen L, Pliakas T, Ayles HJH et al: "Being seen" at the clinic: Zambian and South African health worker reflections on the relationship between health facility spatial organisation and items and HIV stigma in 21 health facilities, the HPTN 071 (PopART) study. 2019, 55:87-99.

29. Opuni M, Bishai D, Gray GE, McIntyre JA, Martinson NAJA, Behavior: Preferences for characteristics of antiretroviral therapy provision in Johannesburg, South Africa: results of a conjoint analysis. 2010, 14(4):807-815.

30. Goossens AJ, Cheung KL, Sijstermans E, Conde R, Gonzalez JG, Hiligsmann MJJoME: A discrete choice experiment to assess patients' preferences for HIV treatment in the rural population in Colombia. 2020, 23(8):803-811.

31. Sijstermans E, Cheung KL, Goossens AJ, Conde R, Gonzalez JG, Hiligsmann MJJoME: A discrete choice experiment to assess patients' preferences for HIV treatment in the urban population in Colombia. 2020, 23(8):812-818.

32. Beach MC, Duggan PS, Moore RDJJogim: Is patients' preferred involvement in health decisions related to outcomes for patients with HIV? 2007, 22(8):1119-1124.

33. Zanolini A, Sikombe K, Sikazwe I, Eshun-Wilson I, Somwe P, Bolton Moore C, Topp SM, Czaicki N, Beres LK, Mwamba CPJPm: Understanding preferences for HIV care and treatment in Zambia: evidence from a discrete choice experiment among patients who have been lost to follow-up. 2018, 15(8):e1002636.

34. Tanle A, Akwasi K-K, Mariwah S, Kobina E-D, Asiedu SO, Lamptey JJGJoG: Preferences for antiretroviral therapy services: qualitative evidence from people living with HIV in Ghana. 2017, 9(1):1-16.

\section{Tables}

Table 1: Socio-demographic characteristics of participants $(n=15), 2022$ 


\begin{tabular}{|ll|}
\hline Characteristic & Percent (number) \\
\hline Age(years) & \\
\hline Mean(SD) & $38.8(3.59)$ \\
\hline Range & 22,72 \\
\hline Gender & \\
\hline Female & $66.67(n=10)$ \\
\hline Male & $33.33(n=5)$ \\
\hline Education & \\
\hline None & $46.67(n=7)$ \\
\hline Primary & $13.33(n=2)$ \\
\hline Secondary and higher & $40.00(n=6)$ \\
\hline Marital status & \\
\hline Married & $26.67(n=4)$ \\
\hline Divorced & $46.67(n=7)$ \\
\hline Never married & $26.67(n=4)$ \\
\hline Employment & \\
\hline Unemployed & $33.33(n=5)$ \\
\hline Unskilled employment & $46.67(n=7)$ \\
\hline Skilled & $20.00(n=3)$ \\
\hline Duration on ART(years) & \\
\hline Mean(SD) & $10.1(1.43)$ \\
\hline Range & \\
\hline
\end{tabular}

n: number; SD: Standard Deviation

Table 2: Categories, themes, and supporting themes for participants' experiences with antiretroviral therapy 


\begin{tabular}{|c|c|c|}
\hline Categories & Themes & Supporting quotes \\
\hline $\begin{array}{l}\text { Perceived/anticipated } \\
\text { stigma }\end{array}$ & \multirow[t]{5}{*}{ Stigma } & $\begin{array}{l}\text { "I take my drug in a hiding place when my child sleeps since } \\
\text { she didn't know about my status. I always come from a } \\
\text { remote area which takes } 1 \text { hour by bus since there is still a } \\
\text { stigma in our community. My social life and work could be } \\
\text { affected if I get service in my locality". (33 years old } \\
\text { female) }\end{array}$ \\
\hline $\begin{array}{l}\text { Enacted stigma from } \\
\text { family }\end{array}$ & & $\begin{array}{l}\text { "No one knows about my HIV status except my wife hencel } \\
\text { didn't experience discrimination from others". (56 years old } \\
\text { male) }\end{array}$ \\
\hline $\begin{array}{l}\text { Enacted stigma from } \\
\text { the community }\end{array}$ & & $\begin{array}{l}\text { "My husband's family members considered me as doing } \\
\text { some evil activity on him. They advised him to stop the HIV } \\
\text { drug and rather go to a traditional healer". (57 years old } \\
\text { female) }\end{array}$ \\
\hline & & $\begin{array}{l}\text { "I have an experience of discrimination by a woman with a } \\
\text { rental dorm. She refused to accept me to rent in her house as } \\
\text { a result of knowing my HIV status". (27 years old female) }\end{array}$ \\
\hline $\begin{array}{l}\text { Enacted stigma from } \\
\text { the healthcare setting }\end{array}$ & & $\begin{array}{l}\text { "I have found a discriminatory action by the gatekeepers. } \\
\text { They [gatekeepers] openly asked me the reason for the entry } \\
\text { to the hospital in front of many people requesting to enter the } \\
\text { hospital during COVID-19 movement restriction". (40 years old } \\
\text { male) }\end{array}$ \\
\hline
\end{tabular}

Waiting time Time

Facility operation

time

Travel time

Frequency of health

facility visit

Time convenience

with school and work

"The main challenge was the long queues here. You may wait for a long time or even postpone to the next day by taking the emergency drugs only". (22 years old male)

"I didn't see the clinic operating on the weekends and before 2:30 in the morning or after 11:30 in the afternoon from Monday to Friday". (40 years old female)

"It takes me 2 hours of travel by bus". (40 years old female)

"I was visiting the facility every month for 7 years". (22 years old male)

"The laboratory test clashes with my education schedule since the facility always tell me to come in the morning where there may be a class in the school". (22 years old female)

"I am still frustrated with disclosing my status to others. I tried to request one of the providers here and she send me my drugs via postal service since I was not able to come on the appointment date due to a clash with my work". (33 years old female)

Costs of

transportation

Costs of drugs for

opportunistic

infections
Costs per

clinic visit
"I come from a remote area which takes 1 hour by bus with 60 birr cost. Since there is still a stigma in our community, I am forced to come here for getting ART service. I may take paying fewer costs for a taxi if lattended in my locality". (33 years old female)

"I pay for the transport and food costs when I come here. But, I do not pay for drugs at this hospital since I have no 
Additional costs

including food

Cost of missing work when seeking care

$\begin{array}{ll}\text { Attitudes and } & \text { Provider- } \\ \text { behaviors of } & \text { patient } \\ \text { healthcare workers } & \text { interaction }\end{array}$

Counseling

Drug and provider availability

Drug availability opportunistic infections". (40 years old male)

"I have discussed with my providers to give me drugs with additional stock at least for 10 days in addition to the usual prescription to avoid work inconvenience". (33 years old female)

"The providers are kind enough to treat us. Some providers have HIV and help us properly. They [providers with HIV] counsel us very helpful in a good manner. It is like knowing about the hungry status of someone by remembering their own hungry experience. I always want to contact them". (33 years old female)

"I found most providers are good at providing service including counseling. But some providers are not showing bright faces, do not give adequate counseling and simply orders to take drugs for 3 or 6 months, and even do not close the door which affects our privacy". (38 years old male)

Providers' availability
"I am satisfied with the drug supply in this hospital". (40 years old female)

"The current service is not bad. It could be better if there are an adequate number of providers here". (22 years old male)

Table 3: Summary of attribute labels, lay terminologies, labels of plausible levels, and illustrative quotes 


\begin{tabular}{|lll}
\hline Attribute label Lay terminology Illustrative quotes & $\begin{array}{l}\text { Labels of } \\
\text { plausible } \\
\text { levels }\end{array}$
\end{tabular}

Participants/others Individual seen at the same appointment visit versus an appointment that includes other patients
"I prefer the individual-based service to avoid disclosing my status when I form groups and take drugs together with the other clients". (40 years old male)

"I prefer the individualized service to avoid clashing with other group members regarding the scheduled time to meet". (72 years old male)

"I prefer the group-based service since we have an opportunity of sharing ideas". (22 years old male)

"I prefer the group-based service since it helps us to support one another by strengthening our social interactions. It also saves our time to come here individually and use our time for our works by taking drugs in the village". (40 years old female)

Individual

Group

ART packaging
Patients stated preferences for ART packaging attributes
"I also expect that the drug package should be changed since the current bottle-based package created discrimination by others as they could easily identify it. The bottle should be changed so that the container can handle many drugs and even we can put it in our pocket to avoid direct advertising act of the current package. (33 years old female)

"I don't have any problem with the drug packaging including the bottle sound. I use festal to carry my drugs since there is a large number of drugs. But, there may be stigma from the community to carry a large volume of drugs for some clients". (57 years old female)

ART room labeling Branding of ART clinics
"I prefer the clear labeling of the ART room to help me in identifying the service delivery room". (72 years old male)

"I prefer the non-posting of the room to avoid discrimination by others while I get into this service room". (22 years old male)
Need for change of drug package
No need for a change of drug package
Clear labeling of ART room

No clear labeling of ART room 
can pick up clients' meds if they are not due for a provider visit

me in drug-taking since there may be some time inconvenience for me to come here". (33 years old female)

"It would be better to have other persons who assist in case of bedridden or paralyzed cases". (72 years old male)

"I prefer to take drugs myself since I will not be happy if another person brings drugs for me that may not be trusted like me". (40 years old male)

"I prefer to come physically here to be checked about my health status and whether the drug is working or not. It should not be thought of simply taking drugs from here". (25 years old female)

Distance from residence to a clinic
The proximity of a health facility to home
"I prefer the clients get the service in a near place. It reduces time, costs for transportation and could help to engage in other work activities". (38 years old male) system in place
No buddy system in place Near

"It is my interest to come from a remote Far place since there is still a stigma in our community while getting service in my locality". (33 years old female)

Drug administration

Drug formulation

\section{Form of drug} administration
"It could be better if there is a vaccination like for the other diseases or an injection that could be used at least for one year". ( 25 years old female)

Novel drug formulation methods for us or drugs with fewer side effects or
"I would be happy if there is a curable drug some additives in the drug that boosts the client's immunity like vitamins". (33 years old female)

"I expect there is a drug that builds our body like proteins in the drug so that you will be fat and similar to other HIV naïve people". (40 years old male)

"The current pill-based drug has side effects on the stomach. It needs reconsideration". (38 years old male)
Pill based

Injection

Drug with additives and/protein
"If possible I prefer if there is a permanent cure for HIV. I would stop visiting this hospital if there is a cure for HIV". (38 years old male)
Drugs with fewer side effects
Frequency of receiving ART
Frequency of routine visits for
"I prefer to come every three months here. It could help me to be checked about my
Effective or curable drug 
"I prefer to come every 6 months per year. But we can come here if we become sick

Every 6 in between the appointment dates". (38 years old female)

"I prefer to come once per year if I am healthy. It avoids transport costs and losing our daily works there at our locality.

I may come at any time here if I have illness in between". (32 years old female)

$\begin{array}{ll}\text { Labeling of ART } & \begin{array}{l}\text { Labeling for } \\ \text { package }\end{array} \\ \text { medicines }\end{array}$

Location of ART service delivery
Preferred place of service for patients
"I don't care about it [labeling of drug package]. There may be some others who may discriminate us [clients with HIV on $A R T]$ when they [others] see the package and read the labeling of $A R V$ drugs on the boxes". (38 years old female)

"I think there should not be the labeling of the drug package to avoid being readable by other people than me like my child. She may search via Google and know about her status". (33 years old female)

"I prefer the facility-based service since there may be a problem that will occur to give service at the community level by the current level of understanding. Providers may break confidentiality to let know others know about my status. ". (33 years old female)

"I prefer the facility-based ART service since the clients could get their providers in time and get appropriate service here. I have a concern there in the community that the providers may not deliver service like the providers in the facility. I may not be at home on the appointment date or I may be not aware of the exact appointment date there and I may create a problem for my providers in this case". (72 years old male)

"I prefer the community-based service since it [community-based service] saves time and lets clients know each other". (57 years old female)

"I would be happy if the service is given at the community level since it avoids a long queue at this hospital and waiting time". (40 years old female)
Medicine labels must be clear

Medicine labels must not be clear

Health facility 
Preferences on involvement in treatment decisionmaking
Preferred participation and roles of patient and provider in treatment decision-making
"I prefer a joint decision to select the model. There could be sharing of each idea by the clients and the providers. There should be an agreement between the two entities. There may be damage if one of the two simply selects the model". ( 25 years old female)

"I couldn't decide my model of choice. The provider should select the appropriate options since he knows the benefits and harms of this approach. The clients shouldn't select the options for them rather the providers should select them". (40 years old male)
Make treatment decisions together with their provider

Providers making treatment decisions entirely by themselves
"I prefer to select the model of my choice since I have a reason to choose from depending on my context. The provider should not decide for me". (72 years old male)
Patients

make treatment decisions on their own
The person providing ART refills
The person who deliver ART refill services
"I prefer the healthcare workers since they have their training. They can give the drugs by knowing the benefits and the harms. But in the case of the HEW or peer leaders, they lacked the appropriate knowledge and even they may give the drugs exchanging of our drugs. I never trust them in this regard". (27 years old female)

"I prefer the healthcare workers since they are trained to identify and manage the problems that I may have by critically evaluating my health status. However, the peer leaders are similar to me in terms of knowledge and couldn't provide drugs for me properly". (38 years old male)

"I prefer the healthcare workers to deliver the ART service. I don't accept the peer leaders distributing our drugs since there is discrimination by the local community perceiving us having a meeting of HIVpositive people there". (40 years old female)

"I prefer the health extension workers to give me drugs since I can go to her without notifying my status". (40 years old male)

"I prefer the peer leaders to bring us our drugs since they know everything and they
Healthcare workers
Health extension workers 
have experience of drug-taking. They

give more empathy to us compared to

health extension and health care workers.

Others couldn't appreciate the context

despite they have trained on the disease

and the drug and give the service by

reading on it". (40 years old female)

Provider's attitude

The spatial organization of service
Staff attitude while in care and treatment
"My choice depends on the provider you get every visit. You may not get the provider that initially hosts you when you come here at your appointment. There is a difference in the kindness of the providers while giving service to us. I had one female provider who treats me with a bright face. Only a few providers are showing their good approach to us. There may be a behavioral problem by providers due to an increased load of clients". (38 years old male)

Choice of separate or integrated service rooms
"I prefer the separate building of the ART room to avoid the associated stigma if the $A R T$ room is available with other service rooms of the health facility". (72 years old male)

"I prefer the service should be given with another service in the same room to avoid HIV status. The providers should treat them accordingly based on the clients' situation instead of a separate service for $A R T^{\prime \prime}$. (33 years old female)

"I would be okay if the service is connected with other services. It is similar to other services in the hospital. I have raised a question for myself why the service room is isolated from other service delivery rooms in the same facility". (22 years old female)
Rude

Nice

Separate service room

Integrated HIV and other services room
Time of facility operation
Health facility's opening days and hours for ART refill
"I prefer the usual working days and hours since it is enough to get the service at that time". (38 years old female)

"I prefer the weekend-based service since I come here freely to take drugs on these days". (22 years old female)

"I prefer the extra facility opening time especially Saturday and Sunday in addition to the usual working hours and days. I am not comfortable with the facility openings before 2:30 in the morning and after 11:30 in the afternoon since we do not come to this hospital at such time". (40 years old female)
Workweek and usual hours

Weekend service only

Workweek and weekend days 
"I choose a 24 hour ART service in this hospital since we may be sick at any time. We get the service in case of difficulty if the facility is opened all days including night". (25 years old female)

Time spent at clinics in ART pickups
Waiting time of registration, consultation, and ART pick- ups
"I would prefer to get service in the shortest time since I am busy either going to work or school. But, if the service demands to wait for more, I would wait here instead". (22 years old male)

"I prefer to wait a long time to learn from my providers about the drug and the related things. I also want to talk with other clients in this hospital. I would be happy when I come here and see other clients taking drugs like me. I become frustrated when I am alone in the rural area of my home". (40 years old female)

The total cost of a The total cost of visit the visit includes transportation, direct medical costs (e.g., consultation or booking fee, lab costs if not available at a public facility, non-ARV drug costs), and costs of food
"I will pay whatever transport cost I have been requested to come here. I have health insurance and do not pay other payments in this hospital". (40 years old female)

"I believe that the free service that is currently being done is a good one. I recommend a balanced payment or a free service for those clients unable to pay for it [service]". (33 years old female)

"I want there should be a free service related to HIV and other illnesses since living nowadays is becoming hard and there are some clients who live in poverty unable to cover the costs of medications". (27 years old female)

"I prefer the service free of charge. There should be a special arrangement from the facility to cover the cost of transportation for those clients who do not generate income like old persons". (25 years old female)

\section{Supplementary Files}

This is a list of supplementary files associated with this preprint. Click to download.

- Additionalfile1.docx

- Additionalfile2.doc 